\title{
Characteristics of Microbes Most Likely to Cause Pandemics and Global Catastrophes
}

\author{
Amesh A. Adalja, Matthew Watson, Eric S. Toner, Anita Cicero \\ and Thomas V. Inglesby
}

\section{Contents}

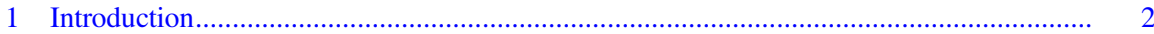

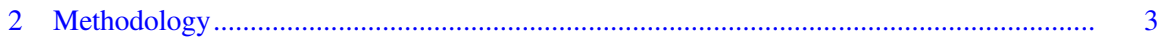

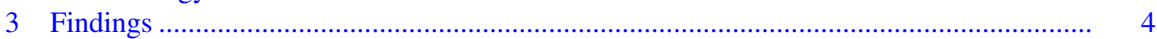

3.1 Specific Microbial Characteristics Are Probably the Most Important Factors Regarding Global Catastrophic Biological Risks ...................................................... 4

3.2 RNA Viruses Are the Class of Microbe that Could Cause a GCBR, Though Other Microbial Classes Could Evolve or Be Engineered in Ways that Pose These Risks

3.3 Bacteria: Broad-Spectrum Antimicrobials Limit Pandemic Potential of Pathogens

3.4 Viral Catalogs Are Scientifically Valuable but Are not Themselves Able to Predict the Next Pandemic

3.5 Increasing Specific Diagnoses of Infectious Disease Syndromes Would Provide Valuable Information and Increase the Chances of Identifying a Pathogen Capable of Causing a Major Epidemic or Pandemic

3.6 Human Factors and/or the Occurrence of Complex Disasters Can Elevate Pathogens to GCBR Levels

4.1 Preparedness for Pandemic and Global Catastrophic Biological Threats Should Acknowledge the Microbial Characteristics that Pose the Greatest Dangers

4.2 Pathogen List-Based Approaches and Precedents Are not Sufficient to Address Pandemic and Catastrophic Biological Risks

4.3 Improving Surveillance of Human Infections with Respiratory-Borne RNA Viruses Should Be a Higher Priority ......

4.4 An Increased Emphasis on Developing a Specific Pipeline of Various Antiviral Agents for RNA Respiratory Viruses-Both Broad Spectrum and Virus-SpecificWould Add Resilience Against Pathogens that Pose Pandemic and Global Catastrophic Biological Risks

\footnotetext{
A. A. Adalja $(\bowtie) \cdot$ M. Watson · E. S. Toner · A. Cicero · T. V. Inglesby Johns Hopkins Center for Health Security, 621 E. Pratt Street Suite 201, Baltimore, MD 21202, USA

e-mail: aadalja1@jhu.edu

Current Topics in Microbiology and Immunology (2019) 424: 1-20 https://doi.org/10.1007/82_2019_176 
4.5 Vaccines Against RNA Respiratory Viruses, Including a Universal Influenza Vaccine, Should Be Pursued with Increased Priority...

4.6 A Clinical Research Agenda for Optimizing the Treatment of Respiratory-Spread RNA Viruses Should Be Funded by Pharmaceutical Companies, Governments, and Medical Device Companies and Pursued by Clinical Centers...

4.7 Special Review Is Warranted for Respiratory-Borne RNA Virus Research that Could Increase Pandemic Risks.

4.8 Pursuing Microbiologically Specific Diagnoses of Infectious Disease Syndromes Should Become More Routine Globally....

\begin{abstract}
Predicting which pathogen will confer the highest global catastrophic biological risk (GCBR) of a pandemic is a difficult task. Many approaches are retrospective and premised on prior pandemics; however, such an approach may fail to appreciate novel threats that do not have exact historical precedent. In this paper, based on a study and project we undertook, a new paradigm for pandemic preparedness is presented. This paradigm seeks to root pandemic risk in actual attributes possessed by specific classes of microbial organisms and leads to specific recommendations to augment preparedness activities.
\end{abstract}

\title{
1 Introduction
}

The recent global experience with severe infectious disease epidemics has triggered much interest in understanding the broader pandemic threat landscape. A substantial proportion of pandemic and biological threat preparedness activities have focused on list-based approaches that were in part based on pandemic influenzas of the past, historical biological weapon development programs, or recent outbreaks of emerging infectious diseases (e.g., SARS, MERS, Ebola) (Centers for Disease Control and Prevention 2017; Casadevall and Relman 2010). But such an approach inherently fails to account for agents not currently known or those without historical precedent. For that reason, preparedness activities that are limited to these approaches may hamper preparedness and lessen resilience.

The purpose of this study was to analyze the characteristics of pathogens that could be capable of causing a global catastrophic biological risk (GCBR). These would be events in which biological agents - whether naturally emerging or reemerging, deliberately created and released, or laboratory engineered and escaped - could lead to sudden, extraordinary, widespread disaster beyond the collective capability of national and international governments and the private sector to control. If unchecked, GCBRs would lead to great suffering, loss of life, and sustained damage to national governments, international relationships, economies, societal stability, or global security (Schoch-Spana et al. 2017).

Given the severe potential public health consequences of pandemic events, there needs to be a vital interest in developing and maintaining a flexible, rapid, and robust 
response capability. Anticipating the forms of microbial threats that might cause future pandemics can help strengthen preparedness and response capacities. This paper proposes a framework for considering future pandemic threats and provides recommendations for how this framework should inform pandemic preparedness.

\section{Methodology}

Review of the published literature and previous reports: The project team surveyed the current biomedical literature on the topic of emerging infectious disease characteristics, the pathogenic potential of microbes, and related topics. The literature review was microbe- and species-agnostic, encompassing all classes of microorganisms and host species. The literature review was accomplished with extensive PubMed searches on these subjects. Relevant US government policy and strategy were reviewed.

Interviews: The project team interviewed more than 120 technical experts who work in and are intimately knowledgeable about this field. Interviewees were drawn from academia, industry, and government. Our goal was to ascertain the experts' views about the essential traits needed for a pathogen to become a GCBR, to contextualize historical outbreaks in light of these traits, and to determine which currently known infectious disease agents possess such characteristics.

Pandemic Pathogen Meeting: The project team completed a preliminary analysis that synthesized the results of our literature review and expert interviews. Those findings were used to design and facilitate a meeting held on November 9, 2017, that included many of those who had been interviewed for this project. The meeting was held at the Johns Hopkins Center for Health Security in Baltimore, MD. The purpose of the meeting was to gain additional insight and input into the project analysis, examine assumptions, and test possible recommendations. Participants included representatives of US and foreign academic institutions, the federal government, and other independent subject matter experts.

This paper is based on the findings of the project and is modification of the project report (Johns Hopkins Center for Health Security 2018). 


\section{Findings}

\subsection{Specific Microbial Characteristics Are Probably the Most Important Factors Regarding Global Catastrophic Biological Risks}

\subsubsection{The Alchemy of a Pandemic Pathogen}

When a pathogen has the capacity to cause a pandemic, it will possess several attributes that other microbes, capable of causing only sporadic or limited human infections, will lack. These traits can be divided into several categories: spread via respiratory transmission; capable of spread during incubation period prior to symptom onset; no preexisting host immunity; and other possible intrinsic microbial characteristics. Many of these characteristics have been captured and are reflected, in equation form, by Casadevall (Casadevall 2017).

\subsubsection{Modes of Transmission}

Microbes have varied routes of transmission, ranging from blood and body fluids to vector-borne to fecal-oral to respiratory (airborne and respiratory droplet). While each mode of transmission is capable of causing large outbreaks if sustained human-to-human transmission is possible and left unchecked, certain modes of transmission are more amenable than others to intervention. For example, the transmission of an infectious disease caused by blood and body fluid transmission can be halted with infection control measures such as gloves or gowns.

Of the various modes of transmission, the respiratory route is the mechanism most likely to lead to pandemic spread. This is chiefly due to the fact that interventions to interrupt this method of spread are more difficult to implement when the simple and universal act of breathing can spread a pathogen. The prolific spread of influenza, pertussis, measles, and rhinoviruses is testament to this fact (Herfst et al. 2017).

By contrast, although pathogens spread by the fecal-oral route, such as Vibrio cholera and the hepatitis A virus, can generate explosive outbreaks, even a modicum of sanitary infrastructure can quench the outbreak.

Vector-borne outbreaks are a special case of a non-respiratory-spread agent. Indeed, the only postulated extinction of a mammalian species by an infectious organism, the Christmas Island rat, was caused by a vector-borne trypanosome (Wyatt et al. 2008). For most of the agents that use this class of transmission, the spread is limited by a geographically and climatologically restricted vector habitat. Humans can protect against vectors, and they can change where they live, but the Christmas Island rat could not. These factors have generally served to limit the pandemic potential of microbes that are spread by vectors.

Exceptions to this general limitation of vector-borne viruses include microbes spread by Anopheles and Aedes mosquitoes. Pathogens spread by these mosquitoes 
have higher pandemic potential, given the geographic breadth of their spread. For example, most of sub-Saharan Africa is hospitable to the malaria-transmitting Anopheles mosquitoes, while residents in $75 \%$ of US counties - as well as half the world's population - are regularly exposed to Aedes mosquitoes that serve as vectors for high viremia flaviviruses and alphaviruses. Such phenomena are borne out by the prolific spread of dengue, chikungunya, and Zika (Sinka et al. 2012; Centers for Disease Control and Prevention 2017).

\subsubsection{Timing of Transmission}

The onset and duration of the period when a person is contagious during an infection also play a major role in spread. Diseases that are contagious during a late stage of infection, when infected people are very sick and therefore have more limited opportunities for spread, may be delimited in their spread. On the other hand, diseases that are contagious prior to symptom development, during the incubation period, or when only mild symptoms are present have greater opportunities for spread as infected individuals are able to conduct their activities of daily living with little or no interruption.

Modeling studies with simulated outbreaks have shown that the presence or absence of this timing of transmission factor can be decisive in whether an outbreak can or cannot be controlled. If a microbe is contagious before a person is seriously ill while the disease is still incubating, then there is higher potential for pandemic spread. Historical examples reinforce this idea, as the only human infectious disease to be vanquished from the planet-smallpox - was one that was not contagious during the incubation period (Fraser et al. 2004). By contrast, a microbe such as the influenza virus, which is contagious prior to symptom development and has a wide range of clinical severity, is able to infect widely and is not amenable to control (Brankston et al. 2007).

\subsubsection{Host Population Factors and Intrinsic Microbial Pathogenicity Characteristics}

Microbial pathogenicity cannot, in reality, be separated from host characteristics. As elucidated by Pirofsky and Casadevall's host damage framework, disease is a complex interplay between a host immune system and a microbe (Pirofski and Casadevall 2008). In congruity with this paradigm, host features and microbial pathogenicity are discussed together.

For a microbe to cause a GCBR-level pandemic, it will be necessary for a significant proportion of the human population to be immunologically naïve to the agent so that the microbe would have a high number of susceptible humans to infect. Additionally, large quantities of a sufficiently effective countermeasure (vaccine or antimicrobial agent) would not be available. Immunologic naïveté would be expected with a zoonotic pathogen. The microbe, correspondingly, would 
have to possess the ability to evade the host immune response through virulence factors, immunological camouflage, or other features that allow a productive infection to ensue.

Additionally, human receptors that are utilized by a pandemic-causing microbe would likely be widespread in the population, facilitating permissive infection in the majority of humans. Receptors may also provide target organ tropism for the agent, allowing severe disease to occur (e.g., lower respiratory tract and central nervous system).

Case fatality rates (CFRs) need not be inordinately high to cause a GCBR-level event, as evidenced by the $2.5 \%$ CFR reported for the 1918 influenza pandemic - the event closest to an actual human GCBR in the modern era (Taubenberger and Morens 2006). A low but significant CFR adheres to the host density threshold theorem. According to this commonly held theorem, a microbe that kills too many of its hosts will run out of susceptible hosts and be extinguished (Cressler et al. 2016). While this may be true of pathogens that are closely linked to one host species, it is not applicable to sapronotic diseases such as amebic encephalitis and cholera (in certain contexts), which can infect and kill without jeopardizing future transmission or survival. Indeed, many extinction-level amphibian infectious diseases are sapronotic in nature, such as the chytrid disease of salamanders and frogs (Fisher 2017).

Additionally, a GCBR-level event may not confer direct mortality. Reproductive effects (i.e., in the manner of rubella or Zika) or carcinogenic effects (e.g., HTLV-1) could, in many ways, be highly detrimental to the future of humanity, as they could lead to significant curtailment of lifespans and diminishing birth rates, which could ultimately result in significant population collapse (Rasmussen et al. 2017; Tagaya and Gallo 2017).

\subsection{RNA Viruses Are the Class of Microbe that Could Cause a GCBR, Though Other Microbial Classes Could Evolve or Be Engineered in Ways that Pose These Risks}

Given the right context, any microbial organism could evolve or be engineered to be a GCBR. However, the most likely cause of a GCBR presently is a virus, with RNA viruses being the most probable (Woolhouse et al. 2013).

\subsection{Bacteria: Broad-Spectrum Antimicrobials Limit Pandemic Potential of Pathogens}

Historically, bacterially caused infections such as plague have had incredible impacts on the human species (Raoult et al. 2013). However, the development of antibacterial therapies, beginning with the sulfonamides in 1935 and then penicillin 
in 1942, has severely limited the ability of this class of microbes to cause a GCBR-level pandemic. In addition, the relatively slower speed of replication and accumulation of mutations also disadvantages this class over viruses. For example, a human infected with the hepatitis $\mathrm{C}$ virus (an RNA virus) produces trillions of virions per day, whereas the doubling time of Yersinia pestis, the cause of plague, is 1.25 h (Neumann et al. 1998; Deng et al. 2002).

The public health crisis of multiple-drug-resistant bacteria, such as carbapenem-resistant enterobacteriaceae (CRE) and others, is very alarming (Logan and Weinstein 2017). The spread of these bacterial agents, for which few if any treatments exist, threatens the entire practice of modern medicine, from cancer chemotherapy to joint replacement therapy. However, these organisms, which have variable attributable mortality, tend to be unable to efficiently infect human hosts that are not compromised or hospitalized. As such, the risk to the general public is constrained.

Large outbreaks of cholera and plague have represented true public health emergencies in Yemen and Madagascar, but their spread reflects severe infrastructure deficiencies caused by war and supply constraints rather than true global pandemic risk (Qadri et al. 2017; Roberts 2017).

\subsubsection{Fungi: Thermal Growth Restriction Limits Pandemic Potential}

Fungi represent prolific pathogens outside of the mammalian species. Outbreaks of chytrid fungal disease in frogs and salamanders as well as snake fungal disease represent true existential threats to affected species (Fisher 2017). However, fungi are largely thermally restricted, and only limited members of this class of microbes can infect warm-blooded organisms such as mammals (Casadevall 2012). Indeed, a fungal filter is hypothesized to have existed and may be partly responsible for mammalian warm-bloodedness. The success of the mammalian-adapted fungus that causes white-nose syndrome in bats is facilitated by the lower body temperature that occurs during their hibernation (Foley et al. 2011).

Human infections with fungi tend to be severely damaging only in an immunocompromised host. The human innate immune system contends with countless fungal spores that are present in every breath of air. As such, many endemic fungal diseases, such as histoplasmosis or coccidioidomycosis, do not cause harm in the majority of immunocompetent humans infected. Even newly emerging fungi such as Candida auris and Cryptococcus gattii are largely subjected to this limitation (Chowdhary et al. 2017; Centers for Disease Control and Prevention 2010). One of the most widespread fungal outbreaks - the Exserohilum fungal meningitis outbreak - was abetted by direct injection of a contaminated medical product into the spinal region of humans, which is not a usual mechanism of infection (Casadevall and Pirofski 2013).

Without thermal adaptation (which might be feasible with deliberate manipulation), fungi, many of which are sapronotic and do not rely on or need mammalian hosts, will not constitute a pandemic threat to humans. 


\subsubsection{Prions: Select Transmission Characteristics Limit Pandemic Potential}

Prions - transmissible infective proteins - are one of the most fascinating and understudied of infectious agents. These agents, which are responsible for diseases such as kuru and new variant Creutzfeldt-Jakob disease (vCJD, the human form of "mad cow disease") in humans, cause scrapie, chronic wasting disease, and bovine spongiform encephalopathy in other mammalian species (Chen and Dong 2016).

Though highly damaging to humans and other species they infect, prions require specific conditions for spread. New variant Creutzfeldt-Jakob disease was to date the most highly publicized outbreak of a human prion disease; it resulted in 229 human cases tied to the consumption of beef products primarily in England in the 1990s and the 2000s (Hilton 2006). Other modes of transmission of CJD tied to iatrogenic spread via contaminated surgical instruments or cadaveric hormone products ceased once protective measures were put in place (Bonda et al. 2016). Kuru, a geographically restricted prion disease, was spread via human cannibalism in Papua New Guinea, and the outbreak abated once that practice was ended in the 1960s (Liberski et al. 2012).

The transmission characteristics of prion diseases are such that very extraordinary circumstances, on a par with human cannibalism or massive food contamination, must be present for a GCBR-level risk to be present for humans. Additionally, and almost by definition, such an event would be slow-moving (prions were once known as "slow viruses").

\subsubsection{Protozoa: Limited Pandemic Pathogen}

Protozoal organisms have the distinction of being the only infectious disease to have caused the extinction of a mammalian species. The Christmas Island rat, unable to outrun its vector, was felled by a vector-borne trypanosome (T. lewisi) during the early twentieth century on the Australian island (Wyatt et al. 2008). Human forms of trypanosomiasis have not risen to such a level of concern.

Human protozoal infections have exerted tremendous pressure on the species, and it is hypothesized that half of all humans who have lived died of malaria, which still kills approximately half a million humans annually (World Health Organization 2017). However, the development of antimalarial compounds and vector avoidance strategies has proved successful when they are able to be employed appropriately, and they have relegated malaria to a pathogen whose impact is amenable to control. Nonetheless, one aspect of malaria is of particular concern: the development and spread of artemisinin-resistant forms, which render treatment extremely challenging with little to no effective antimalarial agents left for use. Largely confined to specific regions of Asia, such as Cambodia and Myanmar, this organism poses severe treatment challenges and, if artemisinin-resistant forms were to spread to Africa, could represent a continent-wide catastrophic biologic risk (Haldar et al. 2018). 


\subsubsection{Other Microbial Classes with Limited Pandemic Risk}

Ameba, ectoparasites, and helminths all have limited pandemic risk, as they are constrained by pathogenicity, transmissibility, or both. Clonally transmissible tumors - such as the notable devil facial tumor disease in Tasmanian devils - are rare occurrences in humans, with restricted modes of transmission (maternal-fetal and organ transplantation).

Space-adapted organisms (e.g., salmonella that originates on Earth but spends time in the space station before coming back to earth) can exhibit enhanced virulence; however, they still are susceptible to antibiotic treatment and normal control measures: There is no evidence they pose greater epidemic risk than normal salmonella (Wilson et al. 2007). An alien microbe species that is obtained on Mars or meteorites and brought back to earth, one of the focuses of the planetary protection program at the National Aeronautics and Space Administration (NASA), was not deemed by our interviewees and meeting participants to be likely to pose a threat. And if such a species were found, it would be unlikely to be adaptable to an Earthlike planet environment, as adaptations to its home planet's markedly different environments would likely preclude adaptations to Earth. Even though the chances of serious biological risk posed by such a sample return are deemed to be low, there are many uncertainties, and the highest level biocontainment procedures are being considered for specimens that might harbor such non-Earth-based organisms (National Research Council 2009).

\subsubsection{Viruses: Several Factors Contribute to Heightened Pandemic Risk}

Traditionally, viruses have been ranked at the highest level of pandemic risk, and dedicated preparedness efforts often focus solely on viruses. A disproportionate focus on viruses is justified, however, based on several aspects unique to the viral class of microbes.

The high rate of replication of viruses - for instance, over 1 trillion hepatitis C virions are produced per day in a human infection - coupled with the mutability inherent in such short generation times gives viruses an unrivaled plasticity. This plasticity allows for host adaptability, zoonotic spillover, and immune system evasion.

The lack of a broad-spectrum antiviral agent-like ones available for bacterial and even fungal organisms - also confers a special status on viruses. With no off-the-shelf treatment available to contain a viral outbreak, and likely no vaccine, containment efforts, at least in the early stages, will likely need to be made in the absence of a medical countermeasure (Zhu et al. 2015).

There is a strong consensus that RNA viruses represent a higher pandemic threat than DNA viruses (Kreuder Johnson et al. 2015). This assessment is derived from the fact that the stability of RNA as a genomic material is less than that of DNA, giving more genomic pliability to the RNA viruses. DNA viruses such as smallpox 
do challenge this assumption, and concern exists surrounding the related risks of monkeypox viruses, which are increasingly spreading in the absence of a smallpox vaccine campaign (Kantele et al. 2016). As monkeypox outbreaks continue to occur with longer chains of transmission, employing smallpox vaccines in target populations might be considered.

Another aspect of viral characterization is the location of replication. Viruses with greater capacity for widespread have been shown in studies to be more likely to replicate in the cytoplasm of a cell (Pulliam and Dushoff 2009; Olival et al. 2017). This is postulated to be due to the higher affinity a virus must have for a particular type of host in order to be permitted entry into its nucleus, and this greater affinity would limit its zoonotic potential because it would be likely to be strongly tied to its usual host. In general, it is DNA viruses that tend to have a nuclear replication cycle, while RNA viruses have a cytoplasmic cycle. Strikingly, smallpox - a DNA virus with proven ability to cause pandemics - is a cytoplasmic replicator, while influenza - an RNA virus with proven ability to cause pandemics - has a nuclear replication cycle. The exceptions to these rules argue against any overly strict adherence to them.

Other factors that may increase a virus' potential to cause a global catastrophic risk include a segmented genome (as exemplified by influenza viruses), a comparatively smaller genome size, and high host viremia (e.g., vector-borne flaviviruses). For example, the flu virus' segmented genome makes novel genetic assortment an eventuality, while a large genome may prevent nimble mutations. However, with each characteristic it is impossible to find a general rule, as exceptions abound.

Among currently studied viruses, the influenza A viruses are widely judged to pose the greatest pandemic risk based on historical outbreaks and viral characteristics (Silva et al. 2017; Imai et al. 2017). Analysis of influenza risks is made in the Centers for Disease Control and Prevention (CDC)'s Influenza Rapid Assessment Tool (IRAT) which ranks H7N9 as the most concerning influenza virus strain (Centers for Disease Control and Prevention 2017).

There are several viral groups other than the orthomyxoviruses (which include the H7N9 strain of influenza A) that are spread by respiratory routes, possess RNA genomes, and merit enhanced attention: paramyxoviruses (especially these three genera: respirovirus, henipavirus, and rubulavirus), pneumoviruses, coronaviruses, and picornaviruses (especially these two genera: enterovirus and rhinovirus). Based on our analysis and their inherent characteristics, these viral groups are the most likely source of a GCBR-level threat. 


\subsection{Viral Catalogs Are Scientifically Valuable but Are not Themselves Able to Predict the Next Pandemic}

There are efforts under way to construct viral catalogs of as many viruses as possible. The explicit aim of these projects is to reduce the uncertainty of outbreaks by extensively cataloging as many viral species as possible, so that a virus that causes a disease is less likely to be truly unknown. At the meeting and interviews for this project, a number of experts expressed concern that, while efforts to catalog and broadly sequence viruses in the animal world would provide new scientific discovery, we should not expect that it will identify the source of the next pandemic or that it can change the work being done for pandemic preparedness. Broad viral sequencing would uncover many novel viruses. However, the vast majority of discovered viruses will not have the ability to infect humans let alone the prospect of widespread in the population. Only a few viruses possess this ability.

This work should be pursued with the objective of fundamental viral scientific discovery, rather than the goal of near-term improvement in pandemic preparedness.

\subsection{Increasing Specific Diagnoses of Infectious Disease Syndromes Would Provide Valuable Information and Increase the Chances of Identifying a Pathogen Capable of Causing a Major Epidemic or Pandemic}

In the clinical practice of medicine, syndromic diagnosis - that is, making a nonspecific diagnosis, such as "sepsis," "pneumonia," or "viral syndrome," with little to minimal laboratory testing - is the norm. Specific diagnosis (i.e., sending patient samples for definitive laboratory diagnosis) is often eschewed if it does not affect clinical management, is costly, and is not revealed with routine tests, and/or if the patient recovers. This practice has become enshrined not only in resource-poor areas in which access to diagnostic testing may be limited, but also in resource-rich areas, like North America and Western Europe, where specific diagnoses are viewed as superfluous.

However, the yield from pursuing an etiologic diagnosis in infectious syndromes such as atypical pneumonia, sepsis, encephalitis, meningitis, and clinically significant fevers of unknown origin may be considerable, as it will provide important insight into the ongoing torrent of threats posed by the microbial world. By causing an infection with enough severity to come to medical attention, the culpable microbes have already established that they are damage-causing pathogens to humans - a feat that only a sliver of the microbial world can accomplish (Woolhouse et al. 2016). Many of these microbial diagnoses cannot be made through the routinely ordered diagnostics. Therefore, a special effort would need to 
be made to get to a microbial diagnosis. If that were to be done more frequently and at a more strategic level around the world, it would provide an opportunity to develop new situational awareness regarding which microbes are circulating and infecting humans - information that is clinically valuable in its own right and more attuned to uncovering GCBR-level pathogens than broad viral cataloging.

Such efforts should not be limited to exotic "hot spots" of disease emergence but should be practiced in localities that are broadly representative of where these conditions occur. Particular hot spots of emergence due to the presence of unique risk factors may be higher yield overall, but they should not be the sole sites of investigation. Infectious disease emergence can occur anywhere, as evidenced by the $2009 \mathrm{H} 1 \mathrm{~N} 1$ pandemic, which was first recognized as the etiology behind a mild pediatric upper respiratory infection in California and West Nile fever emerging in cases of undifferentiated encephalitis in the New York City metropolitan area in the late 1990s (Centers for Disease Control and Prevention 2009; Nash et al. 2001).

Such a program would have significant cost and infrastructure implications in resource-constrained regions, so it would be most logical to set up sentinel or strategic sites for pursuing this level of microbial diagnosis in ways that are broadly representative. In developed nations such as the USA, these programs are available but underutilized because of lack of awareness or perceived lack of value by clinicians, for whom it will often not likely change therapeutic decisions.

\subsection{Human Factors and/or the Occurrence of Complex Disasters Can Elevate Pathogens to GCBR Levels}

Many participants in the project voiced the view that any microbe's pandemic potential could be substantially enhanced by human factors and poor preparedness, which could exacerbate a pathogen's spread or damage-causing potential.

Specific issues identified included gaps in hospital preparedness, medical countermeasure manufacturing capacity, medical countermeasure manufacturing locations, impacts on critical workforce members, and cascading effects on vital programs such as food production. For example, concentration of intravenous fluid manufacturing plants in Puerto Rico created massive shortages after a hurricane took the plants offline in 2017 (Wong 2017). The inability of hospitals to surge to meet enhanced patient needs for ventilators or ICU beds is another potential constraint.

Human factors could also take the form of mistaken actions that are based on political considerations but are not supported by an evidence-based medical rationale, or scientific mistakes based on human error, such as misidentifying a microbe or misinterpretation of scientific or epidemiologic data. For example, early in the SARS outbreak, mistakes regarding the etiology of the viral agent occurred, and the 2014 West African Ebola outbreaks were initially thought to be cholera, delaying response efforts for months (World Health Organization 2014). 
Some participants in this study were of the view that such factors as these could outweigh any intrinsic property possessed by a microbe or any physiologic vulnerability possessed by a human. Magnification by human error could cause delays in response or awareness, allowing a pathogen to spread wider and deeper into the population and rendering containment more difficult, sowing panic, and severely stressing the healthcare infrastructure of a region. The majority view, however, was that intrinsic microbial characteristics are the main driver of a microbe's ability to cause a pandemic.

\section{Recommendations}

\subsection{Preparedness for Pandemic and Global Catastrophic Biological Threats Should Acknowledge the Microbial Characteristics that Pose the Greatest Dangers}

Pandemic preparedness should place a high priority on preparing for RNA viral threats, given their frequent spread by respiratory route, cytoplasmic replication, and high mutability. Surveillance, science, and countermeasure development programs and efforts should logically allocate significant resources to this class of microbes. Except for influenza and certain coronaviruses, there are not major preparedness efforts being made for other viruses in this class of microbes.

While RNA viruses were at the top of the list of concerns, other classes of microbes, such as bacteria, fungi, and protozoa, should not be completely dismissed given characteristic that pose special concerns.

Cultivating and maintaining expertise in the epidemiology, surveillance, and pathogenicity of all classes of microbes, with explicit incorporation of a One Health approach - which incorporates and integrates information from infectious diseases of plants, amphibians, and reptiles - will help foster the broad capacities needed for emerging pandemic and global catastrophic biological risks.

\subsection{Pathogen List-Based Approaches and Precedents Are not Sufficient to Address Pandemic and Catastrophic Biological Risks}

Pathogen-based lists, both USA and global, based on influenza precedents, historical biological weapon programs, and emerging infectious diseases were responsible for galvanizing early activities in the field of pandemic preparedness and have helped drive many important contributions. But these lists could create a sense of confidence regarding the prediction of future pandemic threats. 
Lists can become frozen in the minds of those in the field and may be viewed as exhaustive rather than as starting points. Additionally, inclusion in lists could also be sought for political (and not epidemiologic) reasons if inclusion carries with it the prospect of enhanced funding for a long-neglected endemic problem.

One of the chief rationales behind this project was to attempt to move away from a strict list-based approach when considering pandemic threats and to develop a framework grounded in the facts of a microbe's biology and epidemiology. We recommend that risk assessment be rooted in the actual traits that confer pandemic or global catastrophic biological risks as opposed to a pathogen's presence on some earlier developed list.

\subsection{Improving Surveillance of Human Infections with Respiratory-Borne RNA Viruses Should Be a Higher Priority}

As respiratory-borne RNA viruses have been identified as possessing heightened pandemic potential, it is important to strengthen surveillance activities around these viruses where they currently exist and establish them where they are not yet in place. Currently, of the respiratory-borne RNA viruses, only influenza and certain coronaviruses receive high priority for surveillance.

While some efforts to understand coronaviruses, in the wake of SARS and MERS, exist, there is no systematic laboratory surveillance of coronavirus infections in humans. Similarly, no such program exists for rhinoviruses, parainfluenza viruses, RSV, metapneumoviruses, and similar viruses. Since this class of viruses is most likely to hold the future pandemic pathogen, constructing an influenza-like surveillance approach that better characterizes the prevalence, patterns, and geographic distribution of these viruses should be a priority.

Such an approach would focus on human infections, characterizing the epidemiology, virologic features, antiviral susceptibility (if applicable), and clinical manifestations in a fashion that mimics the extensive influenza surveillance conducted by the CDC and other international entities. 


\subsection{An Increased Emphasis on Developing a Specific Pipeline of Various Antiviral Agents for RNA Respiratory Viruses-Both Broad Spectrum and Virus-Specific-Would Add Resilience Against Pathogens that Pose Pandemic and Global Catastrophic Biological Risks}

Currently, outside of anti-influenza antivirals, there is only one FDA-approved antiviral for the treatment of respiratory-spread RNA viruses (ribavirin). Of the six FDA-approved influenza antivirals - amantadine, rimantadine, baloxavir, zanamivir, oseltamivir, and peramivir - all target influenza viruses specifically and have no activity outside influenza, with two influenza A-specific agents (amantadine and rimantadine) rendered virtually obsolete because of resistance. The other antiviral agent (inhaled ribavirin) is approved for the treatment of respiratory syncytial virus (RSV) but has very limited use due to poor efficacy and major toxicity concerns for both RSV and parainfluenza viruses.

There are currently no approved antivirals for any other respiratory-spread RNA viruses in the world. Prioritization of antiviral compounds against this group of viruses may lead to acceleration of drug development and (government and nongovernment) incentivizing programs. Such antiviral compounds would have an advantage over many other emerging infectious disease countermeasures: These viruses exact a considerable toll in the form of community infections each year, providing a basis for a traditional pharmaceutical market as well as one for emerging infectious disease.

Pursuing not only broad-spectrum RNA antivirals, but also those specifically targeted to specific viruses such as RSV, would increase the likelihood of yield.

Nontraditional molecules, such as monoclonal antibodies and immunomodulators, should also be investigated for a role in the treatment and prevention of RNA virus respiratory infections (Walker and Burton 2018). Such adjunctive treatments may lead to improved clinical outcomes. To date, only one virally targeted monoclonal antibody is FDA-approved: pavalizumab for prevention in high-risk infants.

\subsection{Vaccines Against RNA Respiratory Viruses, Including a Universal Influenza Vaccine, Should Be Pursued with Increased Priority}

As with the above discussion regarding antivirals, the need for vaccines against respiratory-borne RNA viruses should also be prioritized. Currently, aside from influenza, for which a moderately effective but technically limited vaccine exists, there are no other vaccines for respiratory-borne RNA viruses. Experimental vaccines targeting RSV have made it into late clinical development only to fail. 
Several important initiatives in this realm do exist and could be augmented to move beyond specific targets that have already been recognized. For example, the Coalition for Epidemic Preparedness Innovations (CEPI) has selected a coronavirus (MERS-CoV) and a paramyxovirus (Nipah) for vaccine development incentivizing (Røttingen et al. 2017). Such a program could, in potential future initiatives, select additional vaccine targets from this group of viruses and even encourage the development of broadly protective vaccines against groups of viruses-for example, a vaccine that protects against all four strains of human parainfluenza viruses, both MERS and SARS CoVs, and both Hendra and Nipah viruses.

Additionally, the heightened interest at the National Institutes of Health (NIH) in a universal influenza vaccine in the wake of the moderately severe 2017-18 influenza season should be channeled to provide significantly increased resources to this endeavor (Paules et al. 2017). As certain avian influenza viruses are of the highest threat tier, a universal influenza vaccine (even one that just protects against A strains) could substantially hedge against an influenza virus attaining GCBR status.

\subsection{A Clinical Research Agenda for Optimizing the Treatment of Respiratory-Spread RNA Viruses Should Be Funded by Pharmaceutical Companies, Governments, and Medical Device Companies and Pursued by Clinical Centers}

As was evident during the 2009 influenza pandemic and subsequent influenza seasons, the treatment of influenza is suboptimal, despite evidence-based guidance. The status of the treatment for other respiratory viruses is even less defined.

While there currently is not a robust antiviral armamentarium against these viruses, there are important clinical questions that occur with their treatment that merit further study. For example, what adjunctive therapies are useful? What coinfections may be present? At what stage of illness are rescue oxygenation devices warranted? As many of these viruses are highly prevalent in the community and are frequently encountered by clinicians in both outpatient and inpatient settings, finding answers to these questions would render clinicians more adept at dealing with pandemic versions of these viruses.

With respect to influenza, there is a growing literature on the use of antiviral agents in combination with anti-inflammatory agents such as nonsteroidal anti-inflammatory agents (NSAIDs) and macrolide antibiotics (Hung et al. 2017). Untangling the nuances of these treatment effects in order to develop robust guidance would have an impact on the ability to cope with an influenza-driven GCBR. 


\subsection{Special Review Is Warranted for Respiratory-Borne RNA Virus Research that Could Increase Pandemic Risks}

Because of the higher likelihood that a GCBR-level threat might emerge from the group of RNA viruses with respiratory-spread, special attention to research on these agents is warranted if such research could increase pandemic risks. While much research on this class of viruses would be low risk and managed by appropriate approaches to biosafety, experimentally engineered antiviral resistance, vaccine resistance, or enhanced transmission, for example, would raise major biosafety and biosecurity concerns. The 1977 appearance of the H1N1 influenza A strain was thought to have resulted from laboratory escape (Zimmer and Burke 2009). It is important to understand the kinds of work being performed with these agents and, in particular, to know of experiments that are being done or are being proposed that would result in increased pandemic risks. Those experiments should have their own special review and approval process that is consistent with the risks and assesses the risks and benefits of this work before approval or funding of this work.

\subsection{Pursuing Microbiologically Specific Diagnoses of Infectious Disease Syndromes Should Become More Routine Globally}

As unknown infectious syndromes abound in all locations, and any given infectious syndrome may have as its etiology a potentially unknown or unappreciated microbe, specific diagnosis should be a routine endeavor. Atypical pneumonias, central nervous system infections, and even upper respiratory infections often are treated without any etiologic agent being identified.

As diagnostic technologies and devices improve in breadth, speed, and ease of use, the increasing uptake of these devices will provide a new opportunity to enhance situational awareness of an infectious syndrome in any location where they are deployed. Such devices are currently being used in research projects in the developing world. The more routine use of devices, such as multi-analyte molecular diagnostic devices, has the capacity to provide a fuller picture of the microbiological epidemiology of any given syndrome, illuminating what has heretofore been biological dark matter (Doggett et al. 2016; Kozel and Burnham-Marusich 2017). Coupled with heightened surveillance of respiratory-borne RNA viruses, the ability to capture an early signal of a potential pandemic pathogen will be greatly enhanced.

To date, certain considerations have limited the uptake and use of these devices: cost, perceived lack of clinical impact, and constraints on hospital resources such as isolation beds. Impacts on hospitals might be noted in laboratory testing volume as 
well as costs. However, when these devices are viewed in the context of pandemic preparedness, the cost-effectiveness calculation should change. These considerations could be moderated if they are considered part of a hospital's emergency preparedness activities and not exclusively as clinical (they also have benefit for antibiotic stewardship activities in both inpatient and outpatient settings). In fact, the use of these devices should be considered on a par with mechanical ventilators, vaccines, antivirals, and antibiotics in the context of pandemic preparedness. Pilot projects demonstrating the feasibility of procuring such devices for infectious disease emergency preparedness could be conducted.

\section{Conclusion}

Understanding the microbial characteristics most importantly regarding the risks of pandemic or global catastrophic biological threats can help strengthen pandemic preparedness activities. While RNA viruses pose the greatest risks, there are characteristics of other microbial classes that cause special concerns and are important to consider in scientific research agendas and in public health preparedness efforts. This analysis leads to a series of recommendations related to disease surveillance, antiviral and vaccine development, clinical research, and research oversight. Taken together, assessment of key microbial class characteristics plus the focused actions that follow this assessment can broadly help improve preparedness for pandemic and global catastrophic risks.

\section{References}

Bonda DJ, Manjila S, Mehndiratta P et al (2016) Human prion diseases: surgical lessons learned from iatrogenic prion transmission. Neurosurg Focus 41(1):E10

Brankston G1, Gitterman L, Hirji Z, Lemieux C, Gardam M (2007) Transmission of influenza A in human beings. Lancet Infect Dis 7(4):257-65 (Apr 2007)

Casadevall A (2012) Fungi and the rise of mammals. PLoS Pathog 8(8):e1002808

Casadevall A (2017) The pathogenic potential of a microbe. mSphere 2(1) (22 Feb 2017)

Casadevall A, Pirofski LA (2013) Exserohilum rostratum fungal meningitis associated with methylprednisolone injections. Future Microbiol 8(2):135-137

Casadevall A, Relman DA (2010) Microbial threat lists: obstacles in the quest for biosecurity? Nat Rev Microbiol 8(2):149-154

Centers for Disease Control and Prevention (2009) Swine influenza A (H1N1) infection in two children-Southern California, March-April 2009. MMWR Morb Mortal Wkly Rep 58 (15):400-402

Centers for Disease Control and Prevention (2010) Emergence of Cryptococcus gattii-Pacific Northwest, 2004-2010. MMWR Morb Mortal Wkly Rep 59(28):865-868

Centers for Disease Control and Prevention (2017) Bioterrorism agents/diseases. Available at: https://emergency.cdc.gov/agent/agentlist-category.asp. Accessed 31 Jan 2018 (17 Aug 2017)

Centers for Disease Control and Prevention (2017) Zika virus - potential range in US. Available at: https://www.cdc.gov/zika/vector/range.html. Accessed 31 Jan 2018 (20 Sept 2017) 
Centers for Disease Control and Prevention (2017) Influenza-Summary of influenza risk assessment tool (IRAT) results. Available at: https://www.cdc.gov/flu/pandemic-resources/ monitoring/irat-virus-summaries.htm. Accessed 31 Jan 2018 (23 Oct 2017)

Chen C, Dong XP (2016) Epidemiological characteristics of human prion diseases. Infect Dis Poverty 5(1):47

Chowdhary A, Sharma C, Meis JF (2017) Candida auris: a rapidly emerging cause of hospital-acquired multidrug-resistant fungal infections globally. PLoS Pathog 13(5):e1006290

Cressler CE, McLEOD DV, Rozins C, Van Den Hoogen J, Day T (2016) The adaptive evolution of virulence: a review of theoretical predictions and empirical tests. Parasitology 143(7):915930

Deng W, Burland V, Plunkett G et al (2002) Genome sequence of Yersinia pestis KIM. J Bacteriol 184(16):4601-11 (Aug 2002)

Doggett NA, Mukundan H, Lefkowitz EJ et al (2016) Culture-independent diagnostics for health security. Health Secur 14(3):122-42 (May-June 2016)

Fisher MC (2017) Ecology: in peril from a perfect pathogen. Nature 544(7650):300-301

Foley J, Clifford D, Castle K, Cryan P, Ostfeld RS (2011) Investigating and managing the rapid emergence of white-nose syndrome, a novel, fatal, infectious disease of hibernating bats. Conserv Biol 25(2):223-231

Fraser C, Riley S, Anderson RM, Ferguson NM (2004) Factors that make an infectious disease outbreak controllable. Proc Natl Acad Sci USA 101(16):6146-6151

Haldar K, Bhattacharjee S, Safeukui I (2018) Drug resistance in plasmodium. Nat Rev Microbiol (22 Jan 2018)

Herfst S, Böhringer M, Karo B et al (2017) Drivers of airborne human-to-human pathogen transmission. Curr Opin Virol 22:22-29

Hilton DA (2006) Pathogenesis and prevalence of variant Creutzfeldt-Jakob disease. J Pathol 208 (2):134-141

Hung IFN, To KKW, Chan JFW et al (2017) Efficacy of clarithromycin-naproxen-oseltamivir combination in the treatment of patients hospitalized for Influenza A(H3N2) Infection: an open-label randomized, controlled, Phase IIb/III Trial. Chest 151(5):1069-1080

Imai M, Watanabe T, Kiso $M$ et al (2017) A highly pathogenic avian H7N9 influenza virus isolated from a human is lethal in some ferrets infected via respiratory droplets. Cell Host Microbe 22(5):615-626.e8

Johns Hopkins Center for Health Security (2018) The characteristics of pandemic pathogens. http://www.centerforhealthsecurity.org/our-work/pubs_archive/pubs-pdfs/2018/180510pandemic-pathogens-report.pdf. Accessed 2 Aug 2019

Kantele A, Chickering K, Vapalahti O, Rimoin AW (2016) Emerging diseases-the monkeypox epidemic in the Democratic Republic of the Congo. Clin Microbiol Infect 22(8):658-659

Kozel TR, Burnham-Marusich AR (2017) Point-of-care testing for infectious diseases: past, present, and future. J Clin Microbiol 55(8):2313-2320

Kreuder Johnson C, Hitchens PL, Smiley ET (2015) Spillover and pandemic properties of zoonotic viruses with high host plasticity. Sci Rep 7(5):14830

Liberski PP, Sikorska B, Lindenbaum S et al (2012) Kuru: genes, cannibals and neuropathology. J Neuropathol Exp Neurol 71(2):92-103 (Feb 2012)

Logan LK, Weinstein RA (2017) The epidemiology of carbapenem-resistant enterobacteriaceae: the impact and evolution of a global menace. J Infect Dis 215(suppl_1):S28-S36 (15 Feb 2017)

Nash D, Mostashari F, Fine A et al (2001) The outbreak of West Nile virus infection in the New York City area in 1999. N Engl J Med 344(24):1807-14 (14 June 2001)

National Research Council (2009) Assessment of planetary protection requirements for Mars sample return missions. The National Academies Press, Washington, DC

Neumann AU, Lam NP, Dahari H, Gretch DR, Wiley TE, Layden TJ, Perelson AS (1998) Hepatitis $C$ viral dynamics in vivo and the antiviral efficacy of interferon-alpha therapy. Science 282(5386):103-107

Olival KJ, Hosseini PR, Zambrana-Torrelio C, Ross N, Bogich TL, Daszak P (2017) Host and viral traits predict zoonotic spillover from mammals. Nature 546(7660):646-650 
Paules CI, Marston HD, Eisinger RW, Baltimore D, Fauci AS (2017) The pathway to a universal influenza vaccine. Immunity 47(4):599-603

Pirofski LA, Casadevall A (2008) The damage-response framework of microbial pathogenesis and infectious diseases. Adv Exp Med Biol 635:135-146

Pulliam JR, Dushoff J (2009) Ability to replicate in the cytoplasm predicts zoonotic transmission of livestock viruses. J Infect Dis 199(4):565-568

Qadri F, Islam T, Clemens JD (2017) Cholera in Yemen - an old foe rearing its ugly head. N Engl J Med 377(21):2005-2007

Raoult D1, Mouffok N, Bitam I, Piarroux R, Drancourt M (2013) Plague: history and contemporary analysis. J Infect 66(1):18-26 (Jan 2013)

Rasmussen SA, Meaney-Delman DM, Petersen LR, Jamieson DJ (2017) Studying the effects of emerging infections on the fetus: experience with West Nile and Zika viruses. Birth Defects Res 109(5):363-371

Roberts L (2017) Echoes of Ebola as plague hits Madagascar. Science 358(6362):430-431

Røttingen JA, Gouglas D, Feinberg M et al (2017) New vaccines against epidemic infectious diseases. N Engl J Med 376(7):610-613

Schoch-Spana M, Cicero A, Adalja A et al (2017) Global catastrophic biological risks: toward a working definition. Health Secur 5(4):323-328 (Jul/Aug)

Silva W, Das TK, Izurieta R (2017) Estimating disease burden of a potential A(H7N9) pandemic influenza outbreak in the United States. BMC Public Health 17(1):898

Sinka ME1, Bangs MJ, Manguin S et al (2012) A global map of dominant malaria vectors. Parasit Vectors 5:69 (4 Apr 2012)

Tagaya Y, Gallo RC (2017) The exceptional oncogenicity of HTLV-1. Front Microbiol. 2(8):1425

Taubenberger JK, Morens DM (2006) 1918 Influenza: the mother of all pandemics. Emerg Infect Dis 12(1):15-22

Walker LM, Burton DR (2018) Passive immunotherapy of viral infections: 'super-antibodies' enter the fray. Nat Rev Immunol (30 Jan 2018)

Wilson JW, Ott CM, Höner zu Bentrup K et al (2007) Space flight alters bacterial gene expression and virulence and reveals a role for global regulator Hfq. Proc Natl Acad Sci U S A 104 (41):16299-304 (9 Oct 2007)

Wong JC (2017) Hospitals face critical shortage of IV bags due to Puerto Rico hurricane. The Guardian. Available at: https://www.theguardian.com/us-news/2018/jan/10/hurricane-mariapuerto-rico-iv-bag-shortage-hospitals. Accessed 31 Jan 2018 (10 Jan 2018)

Woolhouse MEJ, Adair K, Brierley L (2013) RNA viruses: a case study of the biology of emerging infectious diseases. Microbiol Spectr 1(1) (Oct 2013)

Woolhouse ME, Brierley L, McCaffery C, Lycett S (2016) Assessing the epidemic potential of RNA and DNA viruses. Emerg Infect Dis 22(12):2037-2044

World Health Organization (2014) Ground zero in Guinea: the Ebola outbreak smouldersundetected - for more than 3 months. Available at: http://www.who.int/csr/disease/ebola/ ebola-6-months/guinea/en/. Accessed 2 Feb 2018

World Health Organization (2017) World malaria report 2017. Available at: http://www.who.int/ malaria/publications/world-malaria-report-2017/report/en/. Accessed 31 Jan 2018 (Nov 2017)

Wyatt KB1, Campos PF, Gilbert MT et al (2008) Historical mammal extinction on Christmas Island (Indian Ocean) correlates with introduced infectious disease. PLoS One (11):e3602

Zhu JD, Meng W, Wang XJ, Wang HC (2015) Broad-spectrum antiviral agents. Front Microbiol 22(6):517

Zimmer SM, Burke DS (2009) Historical perspective - emergence of influenza A (H1N1) viruses. N Engl J Med 361(3):279-85 (16 July 2009). https://www.ncbi.nlm.nih.gov/pubmed/ 19564632 